\title{
Anatomical description of the perforating cutaneous nerve
}

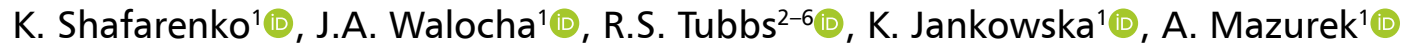 \\ ${ }^{1}$ Department of Anatomy, Jagiellonian University Medical College, Krakow, Poland \\ 2Department of Neurosurgery, Tulane Centre for Clinical Neurosciences, Tulane University School of Medicine, \\ New Orleans, LA, United States \\ ${ }^{3}$ Department of Neurosurgery and Ochsner Neuroscience Institute, Ochsner Health System, \\ New Orleans, LA, United States \\ ${ }^{4}$ Department of Neurology, Tulane Centre for Clinical Neurosciences, Tulane University School of Medicine, \\ New Orleans, LA, United States \\ ${ }^{5}$ Department of Anatomical Sciences, St. George's University, Grenada, West Indies \\ ${ }^{6}$ Department of Surgery, Tulane University School of Medicine, New Orleans, LA, United States
}

[Received: 5 October 2021; Accepted: 12 December 2021; Early publication date: 17 January 2022]

\begin{abstract}
Background: The perforating cutaneous nerve/perforating nerve of the sacrotuberous ligament is rarely observed. It usually arises from the posterior division of the sacral plexus or the pudendal nerve and perforates the sacrotuberous ligament. The anatomy of this nerve and its variants is poorly described in the literature, but there are data indicating its role in pudendal neuralgia.

Materials and methods: Herein, we present an anatomical study of six formalin-fixed cadavers with descriptions of the topography of spinal nerves S2-S4, the pudendal bundle, the perforating cutaneous nerve and the sacrotuberous ligament. Results: We found three perforating cutaneous nerves and described each of them in detail, with measurements of length and width, and point of perforation of the sacrotuberous ligament.

Conclusions: We distinguished three types of perforating cutaneous nerve on the basis of our findings and previous publications; two of the three types were observed in our study. (Folia Morphol 2023; 82, 1: 88-95)
\end{abstract}

Key words: perforating cutaneous nerve, sacrotuberous ligament, pudendal nerve, pudendal neuralgia, anatomy

\section{INTRODUCTION}

The lumbosacral plexus is the largest plexus in the human body. It is made up of ventral rami of the lumbar, sacral and coccygeal spinal nerves. The sacral plexus (SP) begins with the fourth lumbar and ends with the first coccygeal nerve. The surface of the SP adheres to the anterior surface of the piriformis muscle (PM), while its front adjoins the complex of internal iliac vessels. Each spinal nerve, except the pairs from $\$ 4$ to $\mathrm{Co} 1$, is divided into anterior and posterior divisions, which connect to form the nerves of the SP. The SP comprises short and long branches; the short branches are predominantly responsible for innervating the periosteum, joints and muscles such as the piriformis. The long branches are the sciatic nerve, the posterior cutaneous nerve of the thigh, the superior and inferior gluteal nerves, the pudendal nerve $(\mathrm{PN})$, variable the perforating cutaneous nerve (PCN) and the coccygeal nerve (Fig. 1). Bochenek et al. (2008) [1]. The PCN has a diverse anatomical topography: it can arise from the $\mathrm{PN}$, be absent, or emerge from the pudendal plexus independently of the PN [10]. According to Termino- 


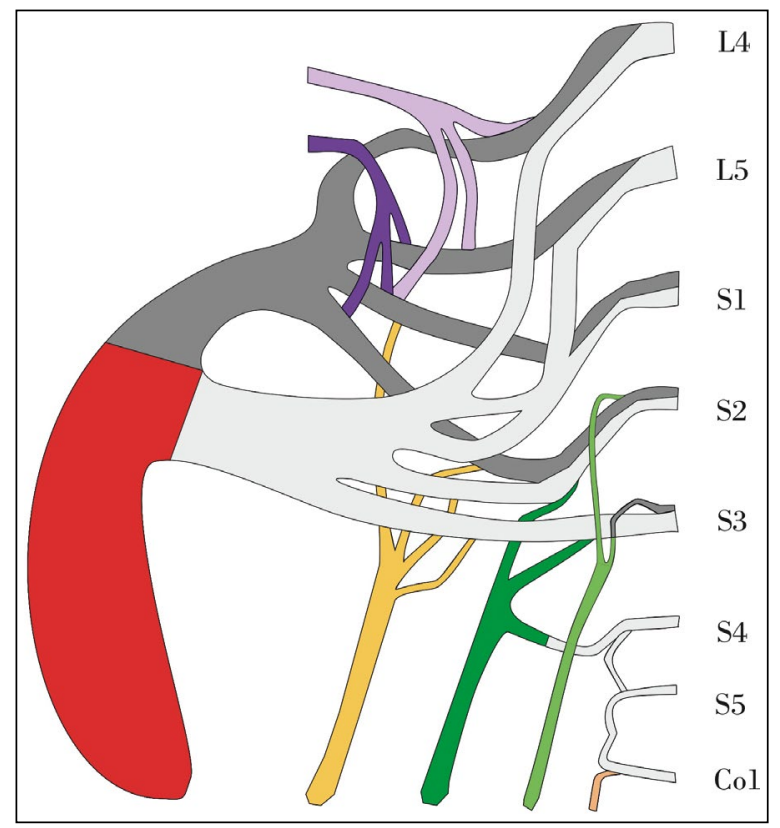

Figure 1. A schematic drawing of the sacral plexus based on "Surgical anatomy of the sacral plexus and its branches", Preface (Fig. 4) [5]. Each spinal nerve, except the pairs from S4 to Co1, is divided into the anterior (light grey) and posterior (dark grey) divisions, which connect forming the nerves of the SP. The sciatic plexus is composed of the sciatic nerve (red), the posterior cutaneous nerve of the thigh (yellow), the superior (light purple) and inferior (dark purple) gluteal nerves. The pudendal plexus incorporates the pudendal nerve (dark green), which is a constant part, and the varying perforating cutaneous nerve (light green). The coccygeal plexus is comprised of the coccygeal nerve (peach).

logia Anatomica 2019, aforementioned name of the nerve determines only the nerve, which arises from the sacral plexus. Therefore, we decided to use the name "perforating of the STL nerve" in a situation where the nerve arises from the PN. All of the long branches of the SP, except the coccygeal nerve [1] and sometimes the PCN [10], extend from the pelvis through the greater sciatic foramen, which is divided into the suprapiriform and the infrapiriform foramina by the PM. The superior gluteal nerve passes though the suprapiriform foramen; the other aforementioned nerves pass through the infrapiriform foramen [1].

The PN usually arises from the ventral branches (anterior divisions) of the S2-S4 spinal nerves and is accompanied laterally by the internal pudendal vessels. It extends from the pelvis under the inferior border of the PM, crosses the ischial spine posteriorly and reenters the pelvis through the lesser sciatic foramen [1]. Subsequently, it reaches the pudendal canal on the medial surface of the ischial tuberosity, where it divides into its terminal branches: the perineal nerve, the dorsal nerve of the penis or clitoris,

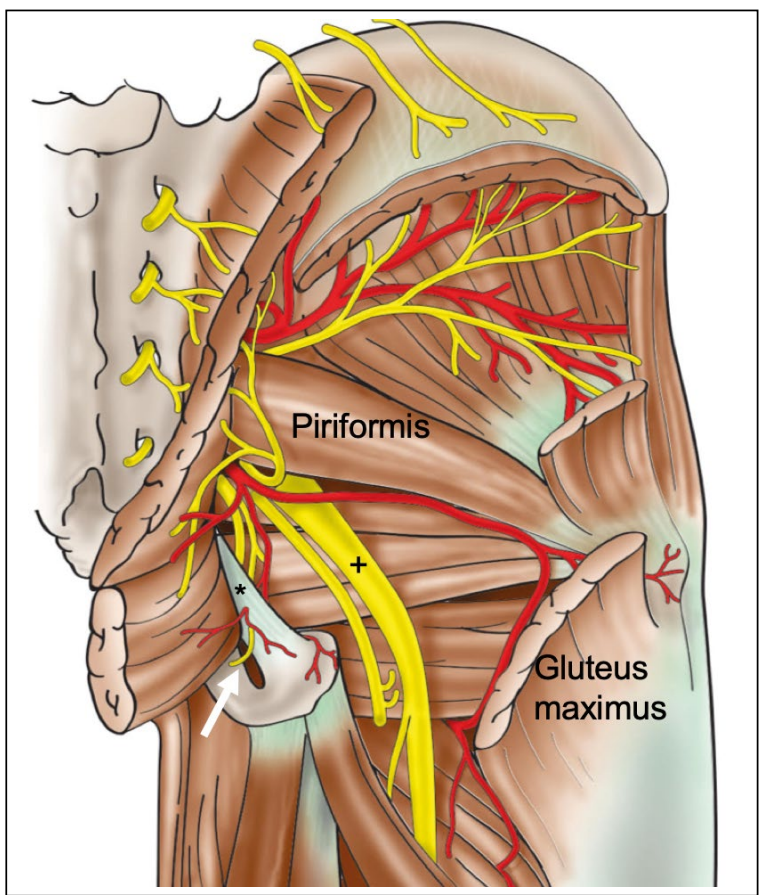

Figure 2. A figure of the perforating cutaneous nerve from "Surgical anatomy of the sacral plexus and its branches", Chapter 6 (Fig. 6.1) [5]. Posterior view of the gluteal region. The perforating cutaneous nerve (arrow) is seen emanating from the sacrotuberous ligament (asterisk). For reference, note the laterally placed sciatic nerve $(+)$.

and the inferior rectal nerve (IRN). The perineal nerve innervates the muscles of the urogenital triangle and the skin on the posterior part of the scrotum or labia majora. The skin of the penis or clitoris is supplied by dorsal nerve of the penis or clitoris. The IRN supplies the external anal sphincter and the mucous membrane of the inferior half of the anal canal and the skin of the anal area $[4,6]$. In $40-56 \%$ of cases the IRN does not enter pudendal canal with the PN but instead reaches the ischioanal fossa $[3,7]$.

In summary, the sensory component of the PN supplies the skin of the pelvic muscles and the genitals except the area around of the mons pubis and the anterior part of the scrotum or labia, whereas the motor component innervates the pelvic muscles and the external genitalia. Moreover, the PN is the only nerve of the SP that carries parasympathetic fibres to the pelvic organs. Its autonomic component is responsible for the processes of urination, defecation and erection. In some cases the PN gives off the perforating of the sacrotuberous ligament (STL) nerve, which most frequently arises in the area surrounding the ischial spine (Fig. 2) [1].

The PN is classified into five types on the basis of the number of its trunks: one-trunked (56.2\%), two-trunk- 
ed (11\%), two-trunked with one trunk as an IRN piercing the sacrospinous ligament (SSL) (11\%), two-trunked with one as an IRN not perforating through the SSL (9.5\%), and three-trunked (12.3\%) $[2,6,8]$. The IRN, which perforates the SSL, is described below.

The aim of this study is to offer a detailed depiction of topography of the PCN, concerning the part between the inferior border of the PM and the inferior border of the STL. The perforating of the STL nerve as a branch of the PN is emphasized. The information is based on dissections of the subgluteal region.

\section{MATERIALS AND METHODS}

There are few data describing the PCN/perforating of the STL nerve, so our research was primarily aimed at on gathering and systematizing all possible information about the subject; the anatomical description was based on our dissections.

Structures required for the study were dissected in embalmed female and male cadavers obtained from the Anatomy Department of the Jagiellonian University Medical College. During our research, 6 cadavers ( 3 female and 3 male) and 12 sacral plexuses were examined through the gluteal approaches. The age, race, weight and cause of death were not provided for scientific purposes.

The gluteal approach was used to expose structures located in front of the gluteus maximus muscle. First, the muscle was cut and the adipose tissue in front of it was removed. The ischial tuberosity was then identified and the STL was separated from the front surface of the gluteus maximus. If the point of perforation through the STL was not found, the dissection was aborted. However, in all cases in the present study the aforementioned point and the PCN/perforating of the STL nerve were found, so the dissections were continued until the pudendal neurovascular bundle, the SSL, and other branches of the PN and nearby structures were displayed. If the PCN/perforating of the STL nerve was present, the ischioanal fossa was additionally dissected in the anteromedial direction to the greatest possible extent of small nerves that branch from PCN/perforating of the STL nerve to determine the range of its innervation. If the PCN arose directly from the SP, as it did in some specimens, part of the sacral bone was removed and the ventral rami of S2-S4 spinal nerves were exposed.

All the steps described below were performed only on cadavers with a PCN/perforating of the STL nerve. The point at which the pudendal neurovascular bundle arose under the inferior border of the PM was documented. The anatomical relationship of the PN

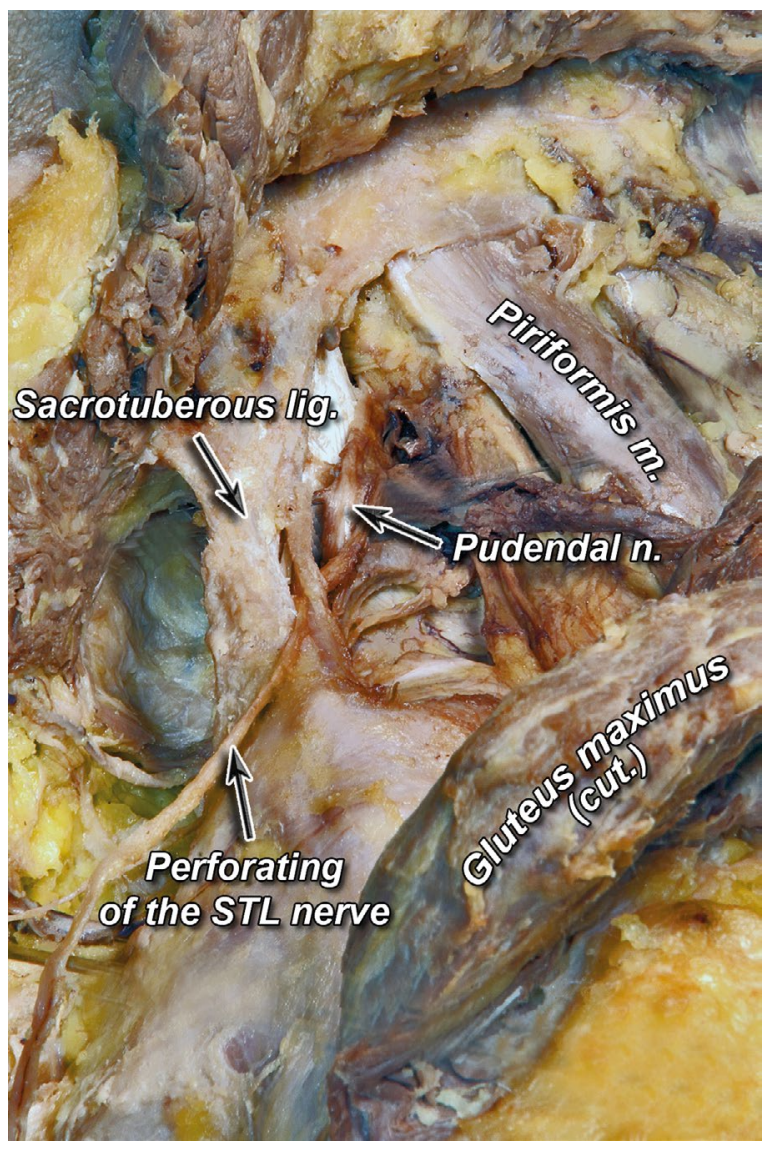

Figure 3. The sacrotuberous ligament (STL) perforated by the right perforating of the STL nerve. The superior view of the right gluteal area shows the perforating of the STL nerve, which arises from the pudendal nerve. It forms a fissure in the ligament during perforation.

to the STL was recorded. The PN, PCN/perforating of the STL nerve, and STL were measured and the anatomical relationship of the PCN/perforating of the STL nerve to the STL was thereby established. The widths and the lengths of the PN, PCN/perforating of the STL nerve, and STL and its fissure, which was formed as a result of perforation, were measured. So were the distances from specifically selected points on the PN to the sacral attachment of the STL, and from the point of origin of the PCN/perforating of the STL nerve to that of the PN under the inferior border of the PM. All measurements were taken 3 times and the median was calculated by the same researcher using the same calliper. Photographs of all cadavers with a PCN/perforating of the STL nerve were obtained.

\section{RESULTS}

During our study, three were found, one of them on the right male gluteal area (perforating of the STL nerve) (Fig. 3) and the other two in female cadavers 


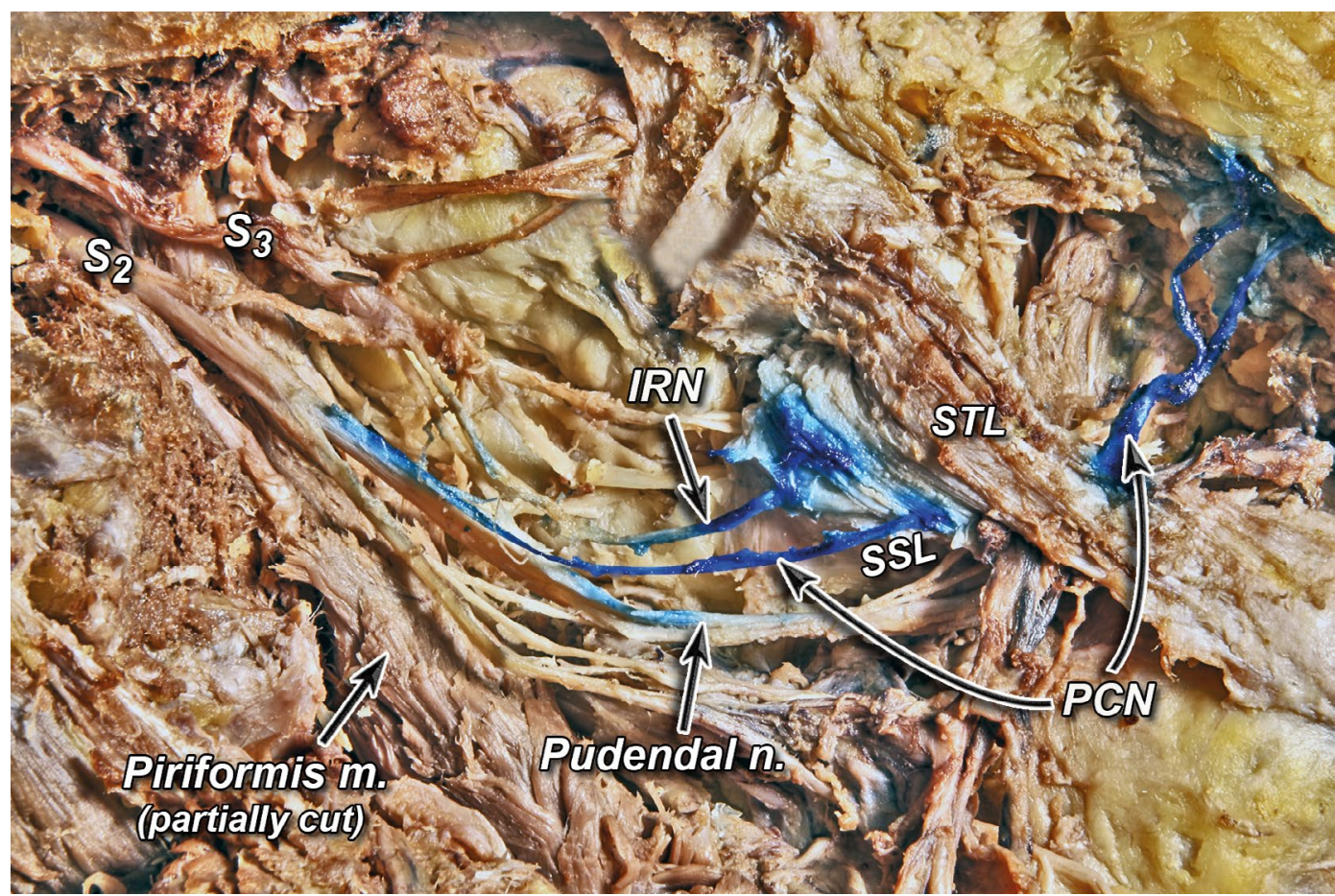

Figure 4. The sacrotuberous ligament (STL) perforated by the left perforating cutaneous nerve (PCN). Two nerves, which perforate the ligaments, were indicated with a bluish tint. The first one perforates the STL and the second one passes through the sacrospinous ligament (SSL); IRN — inferior rectal nerve; $S_{2}$ - ventral branch of the second sacral nerve; $S_{3}$ - ventral branch of the third sacral nerve.

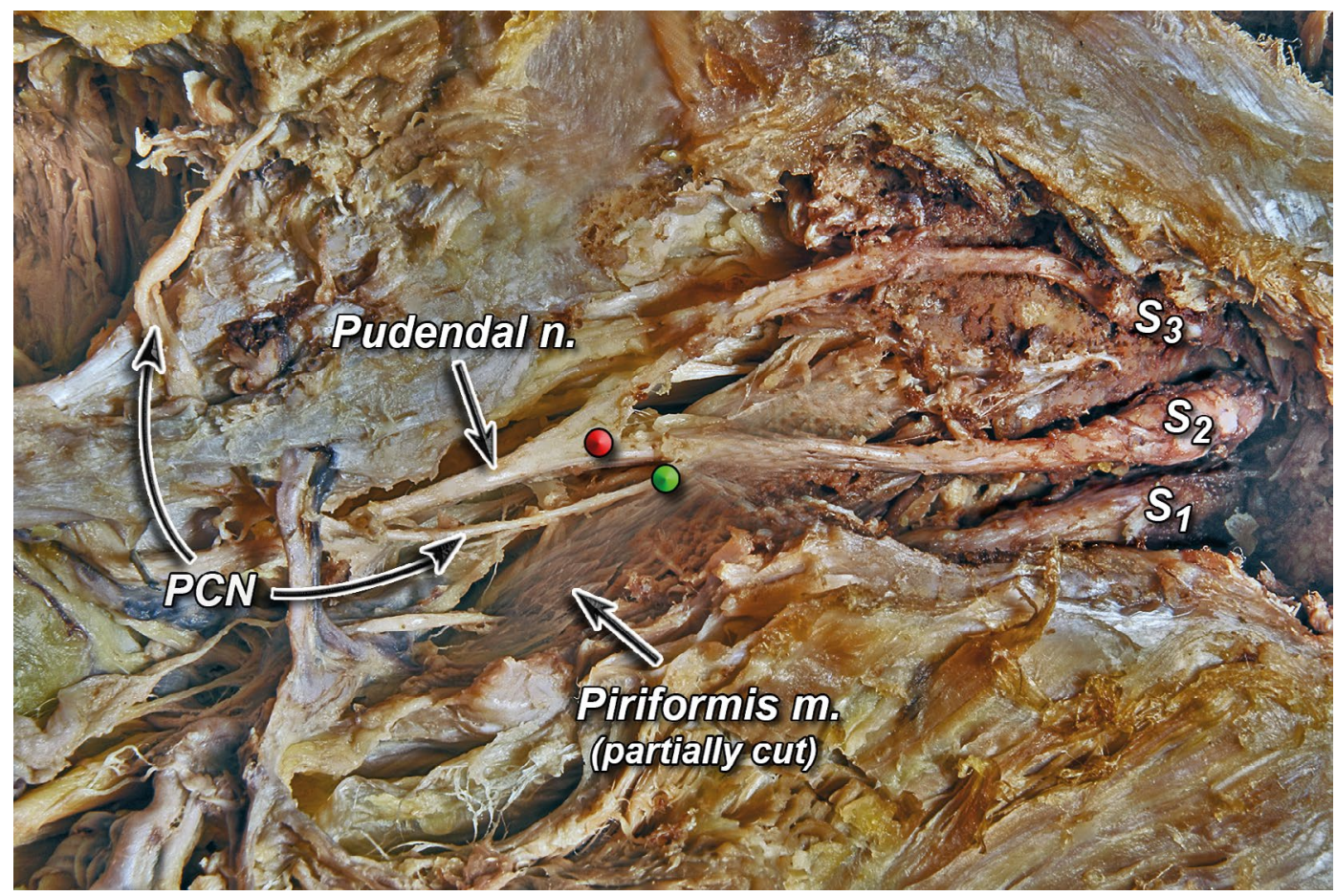

Figure 5. The sacrotuberous ligament perforated by the right perforating cutaneous nerve (PCN). This view shows the PCN above the pudendal nerve. The green pin indicates the origin point of the PCN, while the red pin indicates the origin point of the pudendal nerve; $S_{1}-$ the ventral branch of the first sacral nerve; $S_{2}$ - the ventral branch of the second sacral nerve; $S_{3}$ - the ventral branch of the third sacral nerve.

bilaterally (PCNs) (Figs. 4, 5). Furthermore, we detected a nerve perforating the left SSL in the female cadaver. Its terminal nerve fibres reached the lateral wall of the rectum. From these observations and the 


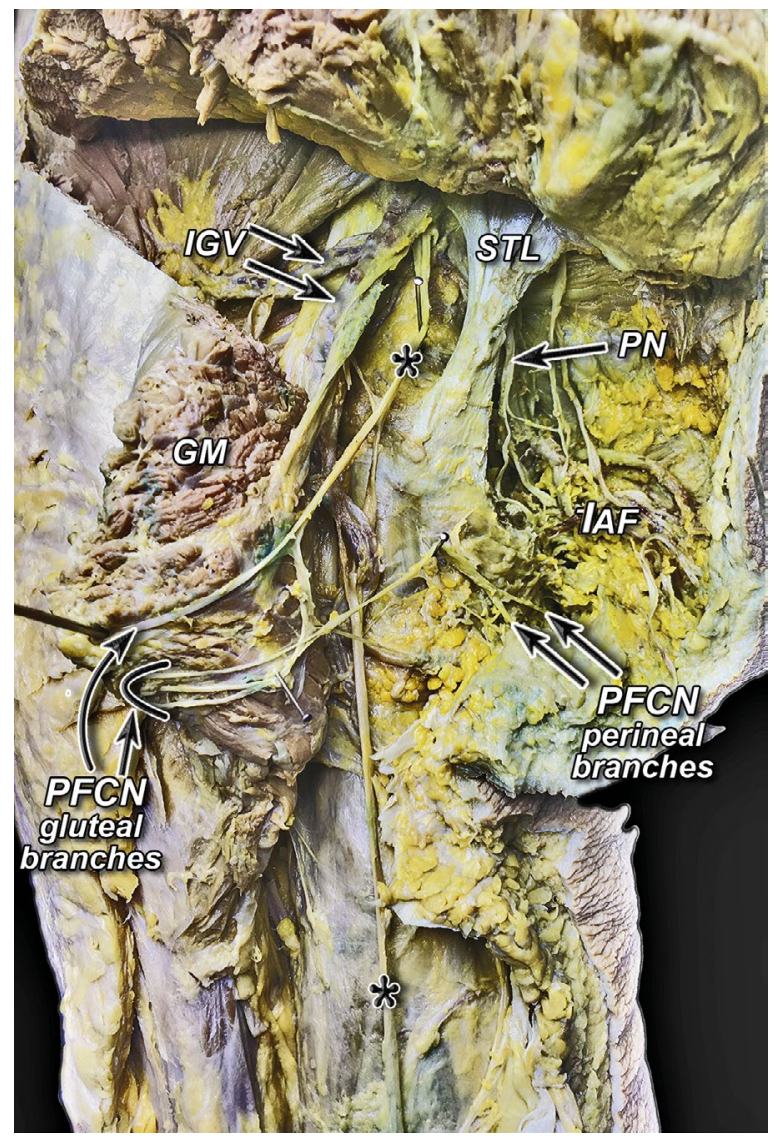

Figure 6. The posterior cutaneous nerve of the thigh with its gluteal and perineal branches; ${ }^{*}$ the posterior cutaneous nerve of the thigh, also known as posterior femoral cutaneous nerve (PFCN); $\mathrm{GM}$ - gluteus maximus muscle; IAF — ischioanal fossa; IGV inferior gluteal vessels; PN — pudendal nerve; STL — sacrotuberous ligament.

classification of the PN in relation to the number of its trunks [6], we concluded that the aforementioned nerve is the IRN (Fig. 4). Considering the specimen with no PCN/perforating of the STL nerves, replacement nerve fibres with the same sensory innervation were found (Fig. 6). These were the gluteal branches from the posterior cutaneous nerve of the thigh.

Our findings show that the PCN/perforating of the STL nerve can arise independently from the ventral branch of the S2 (PCN) or from the PN (perforating of the STL nerve). In 2 cases the nerves arose from the SP (Figs. 4, 5), in 1 case from the PN. In the specimen where the perforating of the STL nerve began as a branch of the PN, the distance between its point of origin and the highest place where the nerve was exposed was $22.38 \mathrm{~mm}$. This means that the perforating of the STL nerve arose at least $22.38 \mathrm{~mm}$ lower than if the PCN had arisen independently from the SP. The origin point of the STL, when it arises from the
PN, is located between the SSL and the STL, which is important as it is the most common location of stricture in pudendal neuralgia cases. The median distance between the point where the PCN/perforating of the STL nerve arose, in the SP and the point of origin of the PN was $9.38 \mathrm{~mm}$ (range: $7.73-11.03 \mathrm{~mm}$ ).

The width of the PCN/perforating of the STL nerve at any point ranges from $0.8 \mathrm{~mm}$ to $1.65 \mathrm{~mm}$ (median $1.10 \mathrm{~mm}$ ). Thus, the $\mathrm{PCN}$ is $2.0-4.6$ times thinner than the PN.

We distinguished two courses for the PCN/perforating of the STL nerve: one is characteristic of the perforating of the STL nerve as a PN branch and the other of nerves arising from the SP. Conventionally, the course of the PCN/perforating of the STL nerve can be divided into three parts: the part before perforation, the perforating part and the part after perforation.

The part of the perforating of the STL nerve before perforation is relatively short. It begins from lateral area of the PN, crosses this nerve posteriorly and almost immediately perforates the inferior part of STL at its superolateral border. If the PCN arises from the SP, the aforementioned part of the nerve is considerably longer. In that case the PCN begins from the lateral or posterior area of the ventral branch of $\mathrm{S} 2$, first accompanying it and subsequently also the $\mathrm{PN}$, later lying medially or laterally to those structures. About halfway from the point of perforation of the STL, the PCN runs obliquely toward the medial side. If the PCN arises from the posterior area, it does not cross the PN but runs between the PN and IRN (medially of the PN and laterally of the IRN) and perforates the STL near the surface of the SSL. However, if it arises from the lateral area, the PCN crosses the PN posteriorly and perforates the STL perpendicular to the course of its fibres.

Regarding the perforating part of the PCN/perforating of the STL nerve, it is important to identify the topographical relationship of the nerve to the ligament. Since the PCN/perforating of the STL nerve perforates the ligament completely differently in different cases, it is essential to discuss each case individually.

The STL perforated by the right perforating of the STL nerve (Fig. 3)

The median length of the perforating of the STL nerve from its departure point to the point of perforation was $19.95 \mathrm{~mm}$. The median length of the STL from the point of perforation to its ischial attachment was $16.02 \mathrm{~mm}$, whereas its median length from the point 
of perforation to the sacral attachment was $65.03 \mathrm{~mm}$. The median width of the STL fibre, which lay laterally from the point of perforation, was $3.08 \mathrm{~mm}$, while the median width of the medial STL fibre was $8.72 \mathrm{~mm}$. After the length and width relationships were calculated, it was concluded that the perforating of the STL nerve perforates the STL around $1 / 5$ lower and $1 / 3$ of its lateral part. The nerve formed a visible fissure, which was $22.75 \mathrm{~mm}$ long, about $2 / 7$ of the total length of the ligament.

\section{The STL perforated by the left PCN (Fig. 4)}

The median length of the PCN from its departure point to the point of perforation was $43.37 \mathrm{~mm}$. The perforation of the STL differed significantly from the one mentioned above. In the former case, the PCN perforated the ligament from front to back, ran through its entire thickness and left a fissure. In the present case, the PCN perforated the ligament vertically, from the top down, entered between its fibres at the superolateral border and ran through its entire width, exiting from its inferomedial border. The PCN did not form a fissure, but "hid" in the ligament forming a canal. It follows from the above that there was no single point of perforation, but rather two: the entry and exit points from the ligament. The total median length of the STL was $69.55 \mathrm{~mm}$. The entry and the exit points were approximately half-way along the STL, and were almost symmetrically located in relation to its midpoint with a deviation about $8-9 \mathrm{~mm}$. The median length of the STL from the entry point to its sacral attachment was $25.96 \mathrm{~mm}$; its median length from the exit point to its ischial attachment was $26.78 \mathrm{~mm}$. There are respectively 8.81 and $7.99 \mathrm{~mm}$ of the distance from the midpoint of the STL $(34.77 \mathrm{~mm})$ to the points mentioned above.

\section{The STL perforated by the right PCN (Fig. 5)}

The median length of the PCN from its departure point to the point of perforation was $42.39 \mathrm{~mm}$, which is about $0.1 \mathrm{~cm}$ less than in the aforementioned case of the nerve in the left buttock. Like, the left $\mathrm{PCN}$, the right $\mathrm{PCN}$ perforated the ligament in an inferomedial direction and entered between its fibres at superolateral border. The difference between the courses of these nerves was that in this case the PCN did not pass through the entire width of the STL but exited it at a point roughly corresponding to half its width. It is worth mentioning that in this case the entry and exit points of the nerve were at the same level in relation to the STL length, in contrast to the left PCN. The total median length of the STL was $64.36 \mathrm{~mm}$. The median length of the STL from the entry and exit points to its sacral attachment was $26.07 \mathrm{~mm}$; its median length from the entry and exit points to its ischial attachment was $38.29 \mathrm{~mm}$. Thus, the PCN perforated the STL at about 2/5 of its upper part.

In summary, the PCN/perforating of the STL nerve can perforate any part of the STL (lower, middle or upper) in the posterior or inferomedial direction by forming a fissure or canal, which may or may not pass the full width of the ligament.

Regarding the part of the PCN after the perforation, the perforating of the STL nerve runs differently from the other two PCNs. After the perforation, it runs on the posterior surface of the STL, heads downwards and medially, crosses the STL attachment to the ischial tuberosity and subsequently runs between the inferior ramus of the ischium and the ischioanal fossa posterior to pudendal canal. Finally, it turns in an anteromedial direction to enter the ischioanal fossa. It is divided into terminal branches that extend to the adipose tissue of the skin and provide sensory innervation to the inferomedial area of the buttock. Moreover, it is possible that the perforating of the STL nerve along with the perineal nerves can innervate a medial area of the perineal skin, since its terminal branches head in that direction. The total median length of the perforating of the STL nerve that arises from the PN is $97.75 \mathrm{~mm}$. The total length of the PCNs, that arise from the ventral branches of the $\mathrm{S} 2$ ranges from 82.16 to $84.32 \mathrm{~mm}$. After the perforation, the aforementioned nerves are divided into terminal branches, which enter between the gluteal maximus muscle and the adipose tissue of the skin and subsequently provide sensory innervation to the inferomedial area of the buttock. However, these branches do not reach the anterior area of the perineal skin. It is worth noting that the mentioned innervation area is similar to the one described by Florian-Rodriguez et al. (2016) [3].

All measurements of the PCN/perforating of the STL nerve are presented in Table 1 and the characteristic traits of its course in Table 2.

\section{DISCUSSION}

The limitation of our study is the insufficient information about PCN/perforating of the STL nerve anatomy in reports in the literature. Nevertheless, our findings correlate with most of the information about the topic in various sources. 
Table 1. Measurements of the perforating cutaneous nerve (PCN)/perforating of the sacrotuberous ligament (STL) nerve

\begin{tabular}{lccc}
\hline Measure & $\begin{array}{c}\text { The right perforating of } \\
\text { the STL nerve (Fig. 2) }\end{array}$ & $\begin{array}{c}\text { The left PCN } \\
\text { (Fig. 3) }\end{array}$ & $\begin{array}{c}\text { The right PCN } \\
\text { (Fig. 4) }\end{array}$ \\
\hline The origin point of the nerve in relation to the origin point of the pudendal nerve & At least $22.38 \mathrm{~mm}$ below & $7.73 \mathrm{~mm}$ above & $11.03 \mathrm{~mm}$ above \\
The width of the nerve & $1.65 \mathrm{~mm}$ & $0.8 \mathrm{~mm}$ & $0.87 \mathrm{~mm}$ \\
The median length of the nerve from its departure point to the perforation point & $19.95 \mathrm{~mm}$ & $43.37 \mathrm{~mm}$ & $42.39 \mathrm{~mm}$ \\
The total length of the nerve & $97.75 \mathrm{~mm}$ & $84.32 \mathrm{~mm}$ & $82.16 \mathrm{~mm}$ \\
\hline
\end{tabular}

Table 2. The characteristic traits of the perforating cutaneous nerve (PCN)/perforating of the sacrotuberous ligament (STL) nerve course

\begin{tabular}{lccc}
\hline Trait & $\begin{array}{c}\text { The right perforating } \\
\text { of the STL nerve (Fig. 2) }\end{array}$ & The left PCN (Fig. 3) & The right PCN (Fig. 4) \\
\hline Does the nerve cross the pudendal nerve? & Yes & No & Yes \\
The direction of the perforation & $\begin{array}{c}\text { Posterior direction } \\
\text { with forming a fissure }\end{array}$ & $\begin{array}{c}\text { Lower-medial direction } \\
\text { with forming a canal }\end{array}$ & $\begin{array}{c}\text { Lower-medial direction } \\
\text { with forming a canal }\end{array}$ \\
The perforation point & $\begin{array}{c}1 / 5 \text { lower and } 1 / 3 \text { of STL lateral part } \\
\text { Lower-medial area of the buttock and } \\
\text { a medial area of the perineal skin }\end{array}$ & $\begin{array}{c}\text { Halfway point of the length of the STL } \\
\text { Lower-medial area of the buttock }\end{array}$ & $\begin{array}{c}\text { 2/5 of STL upper part } \\
\text { Lower-medial area } \\
\text { of the buttock }\end{array}$ \\
\hline
\end{tabular}

Table 3. Difference between publication data and our findings concerning the perforating cutaneous nerve (PCN)/perforating of the sacrotuberous ligament (STL) nerve anatomy

\begin{tabular}{lcc}
\hline & \multicolumn{1}{c}{ Publication data } & Our findings \\
\hline $\begin{array}{l}\text { The presence of the PCN/perforating } \\
\text { of the STL nerve }\end{array}$ & $\begin{array}{c}\text { As a separate unit from S2/S3 with perforation the STL } \\
\text { From the pudendal nerve with perforation the STL } \\
\text { As a separate unit from S2/S3 without perforation the STL } \\
\text { Gluteal branches of the posterior cutaneous nerve } \\
\text { of the thigh (the inferior cluneal nerves) } \\
\text { Perforans coccygeus major nerve }\end{array}$ & $\begin{array}{c}\text { As a separate unit from S2/S3 with perforation the STL } \\
\text { From the pudendal nerve with perforation the STL } \\
\text { of the STL nerve }\end{array}$ \\
\hline
\end{tabular}

First of all, it is worth mentioning that naming of the PCN, which occur in English-language literature, is non-uniform. For example, Tubbs et al. [10] mention the inferior medial cluneal nerve, which is associated with its innervation area. It is also interesting that if this nerve is absent, it is replaced by gluteal branches of the posterior cutaneous nerve of the thigh (Fig. 6). There are reports that the perforans coccygeus major nerve can also replace the PCN, but our findings did not confirm this [10].

Eisler [see 10] found the PCN as a separate unit 22 times in 34 plexuses. He also noted that the PCN can arise from the PN and perforate the STL; this was found three times in the 34 plexuses. Moreover, there are reported cases wherein the PCN does not perforate the STL, instead arising independently from ventral branches of the S2-S3 spinal nerves. If the PCN does not perforate the STL, it will follow the same course as the PN (Table 3$)[2,10]$.
There is no reliable information about the prevalence of the PCN. Our findings show that it occurred in $25 \%(3 / 12)$ of the plexuses examined. Two of the three aforementioned anatomical variations were found during our study. The predominant type $(66.6 \% ; 2 / 3)$ was the independently arising PCN; in $33.3 \%$ the PCN arose as a branch of the PN. In $16.6 \%(2 / 12)$ of the examined plexuses, which had no PCN, there were inferior medialis cluneal nerves (Fig. 6). Another limitation of our study is the fact that not all anatomical variations of the PCN were noted, probably because of the small number of cadavers examined. There is a high probability that the findings would have differed if more plexuses had been studied.

In the studies by Ploteau et al. (2017) [9], the transligamentous course of the PN was mentioned in patients with pudendal neuralgia. It is possible that such a course predisposes to the development of this disease. There were no reports of transliga- 
mentous branches arising from the PN. It is worth mentioning that the target group in this study was quite limited, including only people with pudendal neuralgia, and the symptoms associated with the transligamentous passing of the $\mathrm{PN}$ are so rare $(5 \%$ of nerves including $3 \%$ of perforating the STL) that we found no cases $[8,9]$. The most common location of entrapment of the nerve in patients with pudendal neuralgia is in the space between the SSL and the STL $(74 \%)$. It is most often caused by anatomical variants of the STL and the invasion of ligament fibres into this area. The aforementioned space is a place where the PCN can arise from the PN. Commonly, PN decompression-transposition surgery is performed through the transgluteal approach to ensure constant visual control of all nerves from the gluteal area with a transligamentous course (the PCN perforating the STL, the PN perforating the SSL or STL and the IRN perforating the SSL) [9]. It is worth mentioning that the IRN pierces the SSL in $11 \%$ of the population [6].

There are also reports of PN and PCN injury as a complication following vaginal prolapse repair surgery. Sensory loss results from entrapment of the neural structures by the sutures after cystocele repair surgery. The symptoms concern the skin innervated by the PCN [2].

Florian-Rodriguez et al. [3] marked previously unidentified branches, which are present in $85 \%$ of cases (14 female cadavers). These branches follow different courses from the PCN, but they have the same innervation area. They arise from ventral branches of the S3 or/and S4 sacral nerves and are not associated with the PN or the IRN. Subsequently, they pierce the adipose tissue of the ischioanal fossa ( $\$ 4$ branch) or the inferior border of the gluteus maximus muscle ( $\mathrm{S3}$ branch). The branch from S4 passes between the SSL and the STL without perforating, whereas the branch from S3 perforates the STL. It was concluded that these nerves can be an anatomical variation of the PCN $[3,10]$.

\section{CONCLUSIONS}

The PCN/perforating of the STL nerve is a neural structure that does not always occur, but anatomical knowledge of it is very useful owing to its location. Considering the topographical relationships of the STL, SSL and PCN/perforating of the STL nerve, this nerve is often injured for a variety of reasons. Neuralgia is limited mainly to the inferomedial area of the buttock. Anatomical knowledge of the PCN/perforating of the STL nerve facilitates differentiation from PN neuralgia and helps in making a correct diagnosis.
In addition, it is worth remembering that in surgical practice the STL is tightly connected to the PCN/perforating of the STL nerve, so special care is needed before dissection of the area near the ligament.

\section{Acknowledgements}

The authors sincerely thank those who donated their bodies to science so that anatomical research could be performed. Results from such research can potentially increase mankind's overall knowledge that can then improve patient care. Therefore, these donors and their families deserve our highest gratitude [5].

Also, we acknowledge Jacenty Urbaniak from the Anatomy Department of the Jagiellonian University Medical College for excellent technical assistance during preparation of the figures used in this manuscript.

\section{Conflict of interest: None declared}

\section{REFERENCES}

1. Bochenek A, Reicher M. Anatomia człowieka. Tom V. Wydawnictwo Lekarskie PZWL, Warszawa 2008.

2. Bohrer JC, Chen CC, Walters MD. Pudendal neuropathy involving the perforating cutaneous nerve after cystocele repair with graft. Obstet Gynecol. 2008; 112 (2 Pt 2): 496-498, doi: 10.1097/AOG.0b013e31817f19b8, indexed in Pubmed: 18669778.

3. Florian-Rodriguez ME, Hare A, Chin K, et al. Inferior gluteal and other nerves associated with sacrospinous ligament: a cadaver study. Am J Obstet Gynecol. 2016; 215(5): 646.e1-646.e6, doi: 10.1016/j.ajog.2016.06.025, indexed in Pubmed: 27343565.

4. Gray H. Anatomy of the human body. Bartley.com 2000.

5. Iwanaga J, Singh V, Takeda S, et al. Acknowledging the use of human cadaveric tissues in research papers: Recommendations from anatomical journal editors. Clin Anat. 2021; 34(1): 2-4, doi: 10.1002/ca.23671, indexed in Pubmed: 32808702.

6. Mahakkanukrauh P, Surin P, Vaidhayakarn P. Anatomical study of the pudendal nerve adjacent to the sacrospinous ligament. Clin Anat. 2005; 18(3): 200-205, doi: 10.1002/ ca.20084, indexed in Pubmed: 15768420.

7. Montoya TI, Calver L, Carrick KS, et al. Anatomic relationships of the pudendal nerve branches. Am J Obstet Gynecol. 2011; 205(5): 504.e1-504.e5, doi: 10.1016/j. ajog.2011.07.014, indexed in Pubmed: 21889763.

8. Pirro N, Sielezneff I, Le Corroller T, et al. Surgical anatomy of the extrapelvic part of the pudendal nerve and its applications for clinical practice. Surg Radiol Anat. 2009; 31(10): 769-773, doi: 10.1007/s00276-009-0518-7, indexed in Pubmed: 19495555.

9. Ploteau S, Perrouin-Verbe MA, Labat JJ. Anatomical variants of the pudendal nerve observed during a transgluteal surgical approachin a population of patients with pudendal neuralgia. Pain Physician. 2017; 20(1): E137-E143, doi: 10.36076/2017.1.e137.

10. Tubbs RS, Iwanaga J, Loukas M, Dumont AS, Reina MA. Surgical anatomy of the sacral plexus and its branches. Elsevier, Philadelphia 2021. 